\title{
Diophantine equations concerning various means of binomial coefficients
}

\author{
RICHÁRD RAKAMAZI
}

\begin{abstract}
The main goal of this paper is to show by elementary methods, that there are infinitely many different pairs of binomial coefficients of the form $\left(\begin{array}{c}n \\ 2\end{array}\right)$ such that also their arithmetic, geometric and harmonic means, resp. have the same form. We give all solutions for the arithmetic mean. We also give infinitely many non-trivial solutions for the arithmetic mean of three binomial coefficients satisfying some special conditions. The proofs require the solution of some other interesting Diophantine equations, too. Since the author is also a secondary school teacher, we use elementary methods that mostly can be discussed in secondary school, mainly within the framework of group study sessions. This explains why the means are generally analysed for two terms and for binomial coefficients with "lower" value 2, since further generalizations require substantially deeper mathematical methods which are beyond the frames of this paper.
\end{abstract}

Key words and phrases: diophantine equations, binomial coefficients, arithmetic, geometric and harmonic means of binomial coefficients.

ZDM Subject Classification: F60.

Problem 1. Find all solutions of the Diophantine equation

$$
\frac{\left(\begin{array}{l}
x \\
2
\end{array}\right)+\left(\begin{array}{l}
y \\
2
\end{array}\right)}{2}=\left(\begin{array}{l}
z \\
2
\end{array}\right)
$$

where $y>x \geq 2$.

Solution. If we expand the binomial coefficients and multiply the equation by 16 , we get

$$
4 x^{2}-4 x+4 y^{2}-4 y=8 z^{2}-8 z .
$$


Writing complete squares on both sides, we have

$$
(2 x-1)^{2}+(2 y-1)^{2}=2(2 z-1)^{2} .
$$

This is of the form $A^{2}+B^{2}=2 C^{2}$, which can be reduced to the Pythagorean equation $a^{2}+b^{2}=c^{2}$ by substitutions $A=a-b, B=a+b$, and $C=c$, where $a, b, c>0$ are integers. The general solution is $a=2 u v d, b=\left(u^{2}-v^{2}\right) d$, $c=\left(u^{2}+v^{2}\right) d$, where $u>v>0$ are integers of opposite parity, $\operatorname{gcd}(u, v)=1$, and $d>0$ is an arbitrary integer.

If we substitute these into the original equation, we get

$$
\begin{gathered}
x=\frac{\left|2 u v d-\left(u^{2}-v^{2}\right) d\right|+1}{2}, \quad y=\frac{2 u v d+\left(u^{2}-v^{2}\right) d+1}{2}, \\
z=\frac{\left(u^{2}+v^{2}\right) d+1}{2} .
\end{gathered}
$$

The fractions in this case can be integers only if $d$ is an odd positive integer.

Problem 2. Show that the Diophantine equation

$$
\frac{\left(\begin{array}{c}
x-d \\
2
\end{array}\right)+\left(\begin{array}{l}
x \\
2
\end{array}\right)+\left(\begin{array}{c}
x+d \\
2
\end{array}\right)}{3}=\left(\begin{array}{l}
y \\
2
\end{array}\right)
$$

has infinitely many solutions, where $x \geq d>0$.

Solution. First we verify a Lemma.

Lemma 3. All integer solutions of the equation $x^{2}+6 y^{2}-z^{2}=0$ are given by

$$
\begin{aligned}
& x=\frac{6 u^{2}-24 u v-v^{2}}{\delta} \cdot t \\
& y=\frac{-12 u^{2}-2 u v+2 v^{2}}{\delta} \cdot t \\
& z=\frac{30 u^{2}+5 v^{2}}{\delta} \cdot t
\end{aligned}
$$

where $u$ and $v$ are integers, $v \geq 0, \operatorname{gcd}(u, v)=1, \delta=\operatorname{gcd}\left(6 u^{2}-24 u v-\right.$ $\left.v^{2},-12 u^{2}-2 u v+2 v^{2}, 30 u^{2}+5 v^{2}\right)$, and $t$ is an arbitrary integer. If $t \neq 0$, we get the trivial solution $x=y=z=0$ iff $u=v=0$. The primitive solutions $x=\frac{6 u^{2}-24 u v-v^{2}}{\delta}, y=\frac{-12 u^{2}-2 u v+2 v^{2}}{\delta}, z=\frac{30 u^{2}+5 v^{2}}{\delta}$ are pairwise distinct, and all integer solutions are obtained as the multiples of the primitive solutions. 
Proof. If $z=0$, then obviously $x=y=0$. Suppose that $z \neq 0$. Dividing the equation by $z$ and introducing $X=\frac{x}{z}, Y=\frac{y}{z}$ we get the equation

$$
X^{2}+6 Y^{2}=1 \text {. }
$$

If we could succeed in solving this equation in the set of the rational numbers, then we would also get all integer solutions of the original equation, and vice versa, an integer solution of the original equation gives a rational solution for equation (1) (see [2]). Since $x=1, y=2, z=5$ is an integer solution of the original equation, therefore $X=\frac{1}{5}, Y=\frac{2}{5}$ is a rational solution of equation (1). Equation (1) defines a curve (ellipse) on the plane containing the point $\left(\frac{1}{5}, \frac{2}{5}\right)$. Consider all straight lines on the plane whose slope $m$ is rational, and go through the given point $\left(\frac{1}{5}, \frac{2}{5}\right)$. The equation of the line can be written in the form $Y=m\left(X-\frac{1}{5}\right)+\frac{2}{5}$.

Substituting this into equation (1) and rearranging it, we get the following quadratic equation:

$$
\left(6 m^{2}+1\right) X^{2}+\frac{24 m-12 m^{2}}{5} \cdot X+\frac{6 m^{2}-24 m-1}{25}=0 .
$$

If the discriminant is not zero, i.e. $m \neq-\frac{1}{12}$, the equation has exactly two solutions, one is obviously $X=\frac{1}{5}$, and the other can be obtained for example with the help of Vieta's formulas. We can get the second value of $X$ after a little calculation, $X=\frac{6 m^{2}-24 m-1}{30 m^{2}+5}$. If we substitute this into the equation of the straight line, we get the second value of $Y$, which is $Y=\frac{-12 m^{2}-2 m+2}{30 m^{2}+5}$. If $m$ is rational, then also $X$ and $Y$ are rational numbers. Thus if $m$ runs through the set of the rational numbers, then $X$ and $Y$ exactly provide all rational solutions for equation (1). Intuitively it means, that there exists a one-to-one correspondence between the slopes $m$ and the points with rational coordinates of the ellipse, namely $m$ is the slope of the straight line connecting that point of the ellipse with the point $\left(\frac{1}{5}, \frac{2}{5}\right)$. We can get all rational solutions with this method and we obtain each of them exactly once.

Let $m=\frac{u}{v}$, where $u$ and $v$ are integers, $\operatorname{gcd}(u, v)=1$ and $v \neq 0$. (If $v=0$, then the vertical straight line defines the point $\left(\frac{1}{5},-\frac{2}{5}\right)$ of the ellipse, which also gives us a rational solution.) If we substitute this into the formulae we get $X=\frac{6 u^{2}-24 u v-v^{2}}{30 u^{2}+5 v^{2}}$ and $Y=\frac{-12 u^{2}-2 u v+2 v^{2}}{30 u^{2}+5 v^{2}}$ for the rational solutions of (1), and from here returning to the original equation, we obtain the integer solutions

$$
\begin{aligned}
& x=6 u^{2}-24 u v-v^{2} \\
& y=-12 u^{2}-2 u v+2 v^{2} \\
& z=30 u^{2}+5 v^{2} .
\end{aligned}
$$


For given $u, v, \operatorname{gcd}(u, v)=1$, we divide these integer solutions $x, y, z$ by $\delta=$ $\operatorname{gcd}\left(6 u^{2}-24 u v-v^{2},-12 u^{2}-2 u v+2 v^{2}, 30 u^{2}+5 v^{2}\right)$, and thus obtain the primitive solutions. We can get all integer solutions of the equation $x^{2}+6 y^{2}-z^{2}=0$, if we multiply the primitive solutions by an arbitrary integer $t$.

Now we return to our original problem, to the Diophantine equation

$$
\frac{\left(\begin{array}{c}
x-d \\
2
\end{array}\right)+\left(\begin{array}{c}
x \\
2
\end{array}\right)+\left(\begin{array}{c}
x+d \\
2
\end{array}\right)}{3}=\left(\begin{array}{l}
y \\
2
\end{array}\right) .
$$

If we rearrange the equation and expand the binomial coefficients, we have

$$
(x-d)(x-d-1)+x(x-1)+(x+d)(x+d-1)=3 y(y-1) .
$$

Multiplying the equation by 4 , then adding 3 to both sides, we can create complete squares

$$
[2(x-d)-1]^{2}+(2 x-1)^{2}+[2(x+d)-1]^{2}=3(2 y-1)^{2} .
$$

Let us introduce: $A=2(x-d)-1, B=2(x+d)-1$ and $C=2 y-1$. Since $2 x-1=\frac{A+B}{2}$, we can rewrite the equation into the following form:

$$
A^{2}+\left(\frac{A+B}{2}\right)^{2}+B^{2}=3 C^{2}
$$

Using $A^{2}+B^{2}=2\left[\left(\frac{A+B}{2}\right)^{2}+\left(\frac{B-A}{2}\right)^{2}\right]$, we obtain

$$
3\left(\frac{A+B}{2}\right)^{2}+2\left(\frac{B-A}{2}\right)^{2}=3 C^{2} .
$$

Here $\left(\frac{B-A}{2}\right)^{2}$ is divisible by 3 , and therefore also by 9 , hence dividing by 3 we get the following form:

$$
\left(\frac{A+B}{2}\right)^{2}+6\left(\frac{B-A}{6}\right)^{2}=C^{2}
$$

Let us use the result of the Lemma, but for the sake of simplicity take $t=\delta$. We can see that even with this constraint we can get infinitely many solutions for our Diophantine equation. Using

$$
\begin{aligned}
& \frac{A+B}{2}=6 u^{2}-24 u v-v^{2} \\
& \frac{B-A}{6}=-12 u^{2}-2 u v+2 v^{2}
\end{aligned}
$$




$$
C=30 u^{2}+5 v^{2}
$$

we have

$$
\begin{aligned}
& x=\frac{6 u^{2}-24 u v-v^{2}+1}{2} \\
& d=-18 u^{2}-3 u v+3 v^{2} \\
& y=\frac{30 u^{2}+5 v^{2}+1}{2} .
\end{aligned}
$$

It can be seen from the formulas, that $v$ has to be an odd number. If $x \geq d>0$ then the inequalities

$$
\begin{array}{r}
-18 u^{2}-3 u v+3 v^{2}>0 \\
42 u^{2}-18 u v-7 v^{2}+1 \geq 0
\end{array}
$$

must hold.

We got the second inequality from rearranging $x \geq d$. If we require the stronger inequality $42 u^{2}-18 u v-7 v^{2}>0$ and we have the constraint for $v$ to be a positive odd number, then dividing the inequalities by $v^{2}$, we get quadratic inequalities for $\frac{u}{v}$. In this case we have $-\frac{1}{2}<\frac{u}{v}<-\frac{1}{4}$, from which we can get infinitely many solutions for our original equation even considering the above conditions. If for example $u=-2 t, v=6 t+1$, where $t \geq 1$ is an integer, then we have $x=138 t^{2}+18 t, d=72 t^{2}+42 t+3$ and $y=150 t^{2}+30 t+3$, which if choosing $t=1$ for example, gives the solution

$$
\frac{\left(\begin{array}{c}
39 \\
2
\end{array}\right)+\left(\begin{array}{c}
156 \\
2
\end{array}\right)+\left(\begin{array}{c}
273 \\
2
\end{array}\right)}{3}=\left(\begin{array}{c}
183 \\
2
\end{array}\right) .
$$

Problem 4. Show that the Diophantine equation

$$
\frac{2}{\frac{1}{\left(\begin{array}{c}
x \\
2
\end{array}\right)}+\frac{1}{\left(\begin{array}{c}
y \\
2
\end{array}\right)}}=\left(\begin{array}{l}
z \\
2
\end{array}\right)
$$

has infinitely many solutions, where $y>x \geq 2$.

Solution. Rewriting the equation we get

$$
2 \cdot\left(\begin{array}{l}
x \\
2
\end{array}\right) \cdot\left(\begin{array}{l}
y \\
2
\end{array}\right)=\left(\begin{array}{l}
x \\
2
\end{array}\right) \cdot\left(\begin{array}{l}
z \\
2
\end{array}\right)+\left(\begin{array}{l}
y \\
2
\end{array}\right) \cdot\left(\begin{array}{c}
z \\
2
\end{array}\right) .
$$

We have to solve the equation $2 A B=A C+B C$ with $x(x-1)=A, y(y-1)=B$, and $z(z-1)=C$. Since $C \geq 2$ is even, this equation is equivalent to $A+B \mid A B$. 
Put $d=\operatorname{gcd}(A, B)$, then $A=a d$ and $B=b d$, where $\operatorname{gcd}(a, b)=1$. If we substitute this into the divisibility criterion we get $a+b \mid d a b . \operatorname{Since} \operatorname{gcd}(a b, a+b)=1$, therefore $a+b \mid d$, from which we obtain $d=(a+b) c$. Hence

$$
\begin{aligned}
& A=a c(a+b) \\
& B=b c(a+b) \\
& C=2 a b c .
\end{aligned}
$$

Put $a=c=1$, then we need a $b$ which satisfies

$$
\begin{aligned}
& x(x-1)=b+1 \\
& y(y-1)=b(b+1) \\
& z(z-1)=2 b .
\end{aligned}
$$

If we multiply each equation by 4 and add 1 to both sides, then we can form complete squares on the left hand sides. After these conversions the right hand side of the second equation will automatically be a complete square, thus we need to find suitable positive integers $n$ and $m$ for which $4 b+5=n^{2}$ and $8 b+1=m^{2}$. Substituting these two equalities, we obtain Pell's equation $m^{2}-2 n^{2}=-9$. Any solution $(n, m)$ of this equation, gives an appropriate $b$, since an inspection modulo 8 yields that $n$ and $m$ are odd numbers, therefore their squares are congruent to 1 modulo 8. It is well known (see [1]) that if there exists a solution of this equation among the positive integers, then there are infinitely many solutions. As an illustration, we provide a solution, and will show how to generate a new solution.

We easily get the values $m=3$ and $n=3$, and write this solution in the form $(3-3 \sqrt{2})(3+3 \sqrt{2})=-9$. To create a new solution we shall combine this with one of the infinitely many non-trivial solutions of equation $\left(r^{2}-2 s^{2}=1\right)$, e.g. $1=3^{2}-2 \cdot 2^{2}=(3-2 \sqrt{2})(3+2 \sqrt{2})$. Multiplying the two decompositions we obtain

$$
(3-3 \sqrt{2})(3-2 \sqrt{2})(3+3 \sqrt{2})(3+2 \sqrt{2})=(21-15 \sqrt{2})(21+15 \sqrt{2})=-9 .
$$

Hence also $m=21, n=15$ is a solution. The relevant values $x, y, z$ are $x=8$, $y=56$ and $z=11$ :

$$
\frac{2}{\frac{1}{\left(\begin{array}{l}
8 \\
2
\end{array}\right)}+\frac{1}{\left(\begin{array}{c}
56 \\
2
\end{array}\right)}}=\left(\begin{array}{c}
11 \\
2
\end{array}\right) \text {. }
$$


Problem 5. Show that the Diophantine equation

$$
\sqrt{\left(\begin{array}{l}
x \\
2
\end{array}\right) \cdot\left(\begin{array}{l}
y \\
2
\end{array}\right)}=\left(\begin{array}{l}
z \\
2
\end{array}\right)
$$

has infinitely many solutions, where $y>x \geq 2$.

Solution. After squaring, expanding the binomial coefficients on both sides, multiplying by 64 , and forming complete squares we have

$$
\left[(2 x-1)^{2}-1\right]\left[(2 y-1)^{2}-1\right]=\left[(2 z-1)^{2}-1\right]^{2} .
$$

Let

$$
\begin{aligned}
& A=2 x-1 \\
& B=2 y-1 \\
& C=2 z-1 .
\end{aligned}
$$

Here $3 \leq A<B$, furthermore let's specify the condition $C=\frac{B-A}{2}$. We can show that even with this restriction we get infinitely many solutions for our original Diophantine equation. After substitution and rearrangement, we get the following relation (see [3]):

$$
\left(A^{2}-1\right)\left(B^{2}-1\right)-\left[\left(\frac{B-A}{2}\right)^{2}-1\right]^{2}=0 .
$$

Expanding the brackets, and multiplying by 16, we obtain

$$
\begin{gathered}
16 A^{2} B^{2}-16 A^{2}-16 B^{2}-B^{4}+4 B^{3} A-6 A^{2} B^{2}+4 B A^{3}-A^{4}+8 B^{2}-16 A B+8 A^{2}=0 . \\
-(A+B)^{4}+8 A^{3} B+8 A B^{3}+16 A^{2} B^{2}-8(A+B)^{2}= \\
-(A+B)^{2}\left[(A+B)^{2}-8 A B+8\right]=0 .
\end{gathered}
$$

Since $A+B>0$, we have $(A+B)^{2}-8 A B+8=0$. This equation can be rewritten as

$$
\begin{array}{r}
B^{2}-6 A B+A^{2}+8=0 \\
(B-3 A)^{2}-8\left(A^{2}-1\right)=0 .
\end{array}
$$

Clearly, $4 \mid B-3 A$, thus $B-3 A=4 D$. From this we have Pell's equation $A^{2}-2 D^{2}=1$. If $D<0$ then due to $B>A$ we have $3 A+4 D>A$, therefore 
$A+2 D>0$. However, this is a contradiction since from $-2 D<A$ we get $4 D^{2}<A^{2}=2 D^{2}+1$, i.e. $D^{2}<\frac{1}{2}$. For $D=0$ we would get $A=1$, but $A \geq 3$. Hence $D>0$.

It is well known (see [1]) that Pell's equation $A^{2}-2 D^{2}=1$ is solvable, and all positive integer solutions $\left(A_{n}, D_{n}\right)$ can be obtained from the following recursive formulas: $A_{1}=3, D_{1}=2$, and for $n \geq 1$

$$
\begin{aligned}
& A_{n+1}=3 A_{n}+4 D_{n} \\
& D_{n+1}=2 A_{n}+3 D_{n} .
\end{aligned}
$$

Since $B-3 A=4 D$, therefore $B_{n}=3 A_{n}+4 D_{n}=A_{n+1}$, namely the solutions of equation (2) are $\left(A_{n}, B_{n}\right)=\left(A_{n}, A_{n+1}\right)$. Using the above relations, we can find recursive formulas also for $A_{n}: A_{1}=3, A_{2}=17$ and for $n \geq 1$

$$
\begin{gathered}
A_{n+2}=3 A_{n+1}+4 D_{n+1}=3 A_{n+1}+4\left(2 A_{n}+3 D_{n}\right)= \\
=3 A_{n+1}+8 A_{n}+3\left(A_{n+1}-3 A_{n}\right)=6 A_{n+1}-A_{n} .
\end{gathered}
$$

Since the numbers $A_{n}$ are odd, we only need to show that $C_{n}=\frac{B_{n}-A_{n}}{2}=$ $\frac{A_{n+1}-A_{n}}{2}$ are odd, as well. The first terms of the series are: $A_{1}=3, A_{2}=17$, $A_{3}=99, A_{4}=577$ etc. We show that the terms with even indices are congruent to 1 modulo 4 , while the terms with odd indices are congruent to 3 modulo 4 . We can prove these statements by induction. Supposing the claim holds for the indices $2 k-2$ and $2 k-1$, then $A_{2 k}=6 A_{2 k-1}-A_{2 k-2}=6(4 l+3)-(4 s+1)=4 r+1$ thus we have proven thestatement for $i=2 k$, too. We can proceed similarly for $A_{2 k+1}$. Therefore $A_{n+1}-A_{n}$ are of form $4 m+2$, therefore $C_{n}$ are indeed odd for every $n \geq 1$.

Hence, we have got infinitely many solutions for the equation $\left(\begin{array}{l}x \\ 2\end{array}\right) \cdot\left(\begin{array}{l}y \\ 2\end{array}\right)=\left(\begin{array}{l}z \\ 2\end{array}\right)^{2}$ satisfying $2 \leq x<y$ :

$$
\begin{aligned}
& x=\frac{A_{n}+1}{2} \\
& y=\frac{A_{n+1}+1}{2} \\
& z=\frac{A_{n+1}-A_{n}+2}{4} .
\end{aligned}
$$

Below we summarize some open questions, which have arisen while solving Diophantine equations related to binomial coefficients.

Question 6. 1) Are there (infinitely many) non-trivial solutions for the Diophantine equation $\sqrt{\frac{\left(\begin{array}{c}x \\ 2\end{array}\right)^{2}+\left(\begin{array}{c}y \\ 2\end{array}\right)^{2}}{2}}=\left(\begin{array}{c}z \\ 2\end{array}\right)$ ? 
2) Can all positive integer solutions be given for two terms (with a lower value 2 in the binomial coefficients) for the geometric and harmonic means?

3) Can we find infinitely many or all non trivial solutions for any mean if the number of terms, or the lower value, or possibly both are greater than 2 ?

\section{References}

[1] I. Niven, H. S. Zuckerman and H. L. Montgomery, An Introduction to the Theory of Numbers, fifth ed., John Wiley and Sons Inc., New York, 1991, 351-356.

[2] H. Davenport, The Higher Arithmetic, eighth ed., Cambridge University Press, Cambridge, 2008, 138-139.

[3] A. Schinzel and W. Sierpinski, Sur l' équation diophantienne $\left(x^{2}-1\right)\left(y^{2}-1\right)=$ $\left[\left(\frac{y-x}{2}\right)^{2}-1\right]^{2}$, Elem. Math. 18 (1963), 132-133.

RICHÁRD RAKAMAZI

JÁNOS NEUMANN SECONDARY SCHOOL

RÁKÓCZI STR. 48.

3300 EGER

HUNGARY

E-mail: raka82@gmail.com

(Received August, 2013) 